\title{
Photocatalytic oxidation degradation mechanism study of methylene blue dye waste water with $\mathrm{GR} / \mathrm{iTO}_{2}$
}

Xi-quan WANG ${ }^{1}$, Song-fu HAN ${ }^{1}$,Qing-wen Zhang ${ }^{2}$, Nan Zhang ${ }^{1}$, and Dan-dan ZHAO ${ }^{1}$

${ }^{1}$ Dept. of Environmental Engineering, School of Chemical Engineering, Liaoning University of Science and Technology, Anshan 114051, China

${ }^{2}$ Dept. of Angang Group Engineering Technology, Anshan 114051, China

\begin{abstract}
Graphene/titania multiplex photo-catalyst was prepared through hydrothermal method. Methylene blue with initial concentration of $20 \mathrm{mg} / \mathrm{L}$ in aqueous solution was treated by photocatalytic oxidation and the degradation process was investigated by UV/Vis spectrum, FTIR and GC-MS Spectra analysis. The results indicated that the conjugate structure of N-S heterocyclic compound was broken and aromatic ring was oxidized to open the ring. The methylene blue molecules were finally mineralized to $\mathrm{H}_{2} \mathrm{O}$ and $\mathrm{CO}_{2}$ in the photocatalytic degradation process.
\end{abstract}

\section{Introduction}

Dye wastewater is hard to degrade due to its high content of organic compounds, complexity of the components and high chroma ${ }^{[1]}$. In view of the difficulty in dealing with dye wastewater by the traditional methods, a lot of research work and trials have been done to seek appropriate technology. Photocatalytic oxidation is regarded as a future advanced oxidation technology for the removal of toxic organic dye from water. Among various semiconducting materials, most attention has been focused on $\mathrm{TiO}_{2}$ because of its characters of environmental-protecting, sound stability, non-secondary-pollution and effective degradation ${ }^{[2]}$. The hydroxyl radicals caused by the excitation of $\mathrm{TiO}_{2}$ under UV irradiation could oxidize and mineralize organic pollutants into $\mathrm{H}_{2} \mathrm{O}$ and $\mathrm{CO}_{2}$. However, the photocatalytic activity of $\mathrm{TiO}_{2}$ is unsatisfactory because of the recombination of light-generated electron-hole pairs and the agglomerate phenomena ${ }^{[3]}$. It has been proved that the $\mathrm{TiO}_{2}-\mathrm{C}$ compound materials have more outstanding catalyzed effect ${ }^{[4-5]}$. Graphene is regarded as a good carrier for photocatalyst owing to its large specific surface, fast-paced electronic conduct and large mechani-

-cal strength. The unique physical properties could be favorate to separate electron-hole pairs and avoid agglomeration that in turn leading to the enhanced photocatalytic activity ${ }^{[6-7]}$. Methle-

-ne blue is a widely used organic toxic dye in a range of different fields, which can cause environmental contaminations to the harm environment and the human health. The adsorption characteristics of MB on various adsorbents have previously been extensively investigated, nevertheless, the degradation mechanism of methylene blue in photocatalytic oxidation process is still poorly known owing to its complex chemical structure ${ }^{[8-9]}$. In this paper, Graphene/titania $\left(\mathrm{GR} / \mathrm{TiO}_{2}\right)$ multiplex photocata

lyst was prepared through hydrothermal method. Following previous studies, the intermediate products during the photocatalytic reaction were analysised with UV/Vis, IR and GC-MS spectra to propose the detailed degradation pathways between photocatalytic oxidation and methylene blue solution.

\section{Materials and methods}

\subsection{Dye materials}

The cationic dye, methylene blue (analytically pure) was purchased from Sinopharm chemical reagent Co. LTD. Methylene

blue, 3,7-bis(dimethylamino)-phenothiazin-5-ium chloride, is a heterocyclic aromatic chemical compound with the molecular formula $\mathrm{C}_{16} \mathrm{H}_{18} \mathrm{~N}_{3} \mathrm{SCl}^{[10]}$. The stock solution was prepared by dissolving accurately weighted dye in deionized water to the concentration of $1000 \mathrm{mg} / \mathrm{L}$. The working solutions were obtained by diluting the dye stock solution to the concentration of $20 \mathrm{mg} / \mathrm{L}$. The $\mathrm{pH}$ variation during the course of reaction was not significant, so the solutions were conducted at natural conditions.

*Corresponding author :Tel: 86 18340797937; E-mail:2920816646 @qq.coma 
<smiles></smiles>

Structural formula of methylene blue

\subsection{Chemical reagent}

Butyl titanate, nitric acid, glacial acetic acid, Anhydrous ethanol, phosphoric anhydride, sulphuric acid, graphite powder, methylene blue, potassium persulfate, potassium permanganate, The chemical reagents listed are analytically pure.

\subsection{Photocatalytic oxidation experim-}

-ents

Graphite oxide was prepared by modified Hummers method $^{[11]}$. A predetermined amount of graphite oxide was slowly added into $\mathrm{TiO}_{2}$ sol which was prepared by sol-gel method. Stirring the mixture was continued for about $2 \mathrm{~h}$, and then the solution was aged at room temperature. Then the gel was hydrothermally treated at $200^{\circ} \mathrm{C}$ for $12 \mathrm{~h}$. After this hydrothermal process, the product was washed by deionized water to neutrality, then dried at $70^{\circ} \mathrm{C}$. Finally, the $\mathrm{GR}-\mathrm{TiO}_{2}$ photocatalyst was obtained. The preparation method of $\mathrm{TiO}_{2}$ was similar to that of $\mathrm{Gr}^{-} \mathrm{TiO}_{2}$, except there was no introduction of graphene oxide into the preparation system.

The Photocatalytic oxidation experiments were carried out in photocatalytaic reactor at room temperature. The reactor consists of ultraviolet lamps (main wavelength is $254 \mathrm{~nm}$ ) and magnetic stirrers. In our study, a volume of $100 \mathrm{ml}$ of the aqueous solution of methylene blue with the concentration of $20 \mathrm{mg} / \mathrm{L}$ was introduced in reactor with $100 \mathrm{mg}$ photocatalyst, and then the mixture was stirred for $30 \mathrm{~min}$ in the darkness, after which the reaction suspension was irradiated under UV light. The reaction mixture was stirred vigorously using magnetic stirrer for during the entire reaction time. Samples were collected at appreciate intervals and were centrifuged to remove catalyst particles with high-speed centrifugal.

\subsection{The analysis method}

The X-ray diffractometer (XRD) was carried out to identify the crystal phase. The surface morphology of samples was determined by scanning electron microscopy (SEM) and transmission electron microscopy (TEM). A UV/VIS spectrometer recording the spectra over the $200 \mathrm{~nm}-800 \mathrm{~nm}$ range was used to measure methylene blue solution samples at 10min interval during photocatalytic oxidation. On the other hand, the samples were dried completely and then pressed as $\mathrm{KBr}$ pellets to acquire the FTIR spectra by an FTIR spectrometer from 400 to $4000 \mathrm{~cm}^{-1}$. Intermediate compounds of methylene blue solution were also identified by a GC-MS system. Before analyzing with the GC-MS system, the products were extracted using trichloromethane in a separating funnel for three times.

\section{Results and discussion}

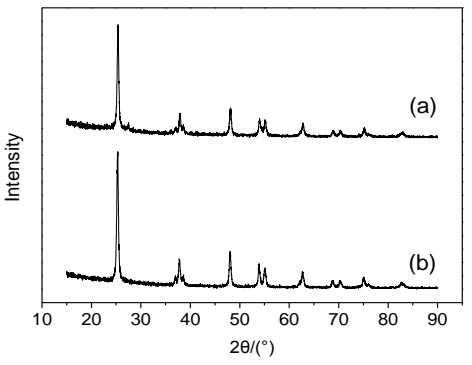

Fig.1 XRD spectras of $\mathrm{GR} / \mathrm{TiO}_{2}$ (a) and $\mathrm{TiO}_{2}$ (b)

Figure 1 shows the XRD patterns of $\mathrm{GR} / \mathrm{TiO}_{2}$ (a) and $\mathrm{TiO}_{2}$ (b). As shown in Fig. $1,25.3^{\circ}, 37.8^{\circ}, 48.0^{\circ}, 53.9^{\circ}$, $55.0^{\circ}, 62.8^{\circ}, 68.8^{\circ}, 70.3^{\circ}, 75.1^{\circ}, 76.1^{\circ}$ and $82.8^{\circ}$ were all characteristic peaks of anatase, indicating that the $\mathrm{TiO}_{2}$ in GR/ $\mathrm{TiO}_{2}$ were anatase crystal which had better photocatalytic active relative to brookite and rutile. Combared Fig.1(a) with Fig.(b), the XRD spectras of $\mathrm{GR} / \mathrm{TiO}_{2}$ (a) and $\mathrm{TiO}_{2}$ (b) were basically the same, illustrating that the peaks of GR were covered and crystal phase of $\mathrm{TiO}_{2}$ remained unchanged.

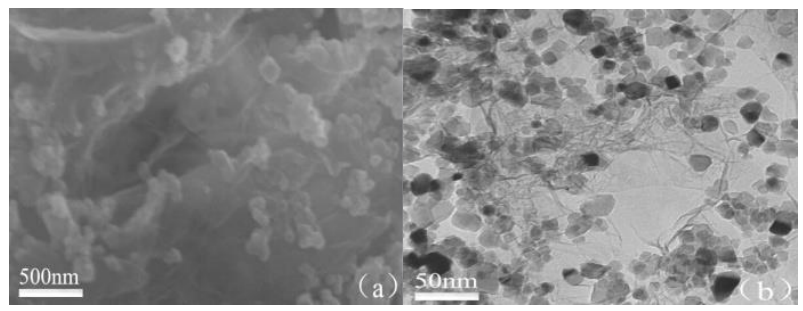

Fig.2 SEM (a) and TEM (b) photo of microstructure of

$$
\mathrm{GR} / \mathrm{TiO}_{2}
$$

Figure 2 shows the SEM (a) and TEM (b) images of GR$\mathrm{TiO}_{2}$. As shown in Fig.2(a), the $\mathrm{TiO}_{2}$ powers had uniform loaded into GR that had high specific surface area. The images indicated that the $\mathrm{TiO}_{2}$ in composite catalyst was approximately $25 \mathrm{~nm}$ in diameter and scattered on the film-shaped GR. With loose agglomeration and favorable dispersibility, that is, dye molecules and catalysts contact area was increased, and resulting in the enhancement of photocatalytic activity.

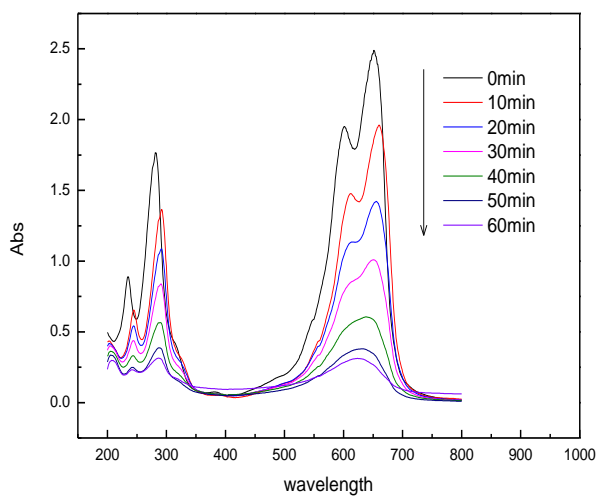

Fig.3 The UV - Vis absorption spectra of methylene blue 
（ $\mathrm{t}=0 \sim 1 \mathrm{~h})$ in photo-catalytic oxidation process

Fig.3 shows the UV-Vis absorption spectra of methylene blue solution at different photocatalytic oxidation reaction time. For the UV-Vis absorption spectra, $612 \mathrm{~nm}$ and $665 \mathrm{~nm}$ in the visible region is the characteristic peak of large conjugated system of N-S heterocycle group. The $291 \mathrm{~nm}$ characterizes phenothiazine structure and the peak of $245 \mathrm{~nm}$ in UV region belong to aromatic and polycyclic aromatic. Methylene blue is a chromogenic agent whose $\mathrm{N}-\mathrm{S}$ heterocycle group attached to benzene includes lone pair electron, in which sulfhydryl group is the main chromophoric group. According to the UV-Vis absorption spectra during reaction time, the strong absorption peaks in the visible region decreased obviously, in view of the absorption peaks become sharper with the extending of the conjugated unsaturated system, indicating that conjugated structure of heterocycle was destroyed. The results in Fig. 3 stated that the destruction of the conjugate structure was almost synchronization during the processing of decolorization. At the same time, the characteristic absorption peaks between 200 and 400nm were gradually reduced, which stated that phenothiazine structure and benzene were attacked by the hydroxyl radicals and oxidized to open the ring. Thus, it is concluded that strong oxidizing substance hydroxyl radicals which generated by ultraviolet dissociating $\mathrm{TiO}_{2}$ directly broke directly unsaturated bonds of conjugated system and change the structure of dye molecule, then the intermediate would be decomposed into small molecules during photocatalytic oxidation.

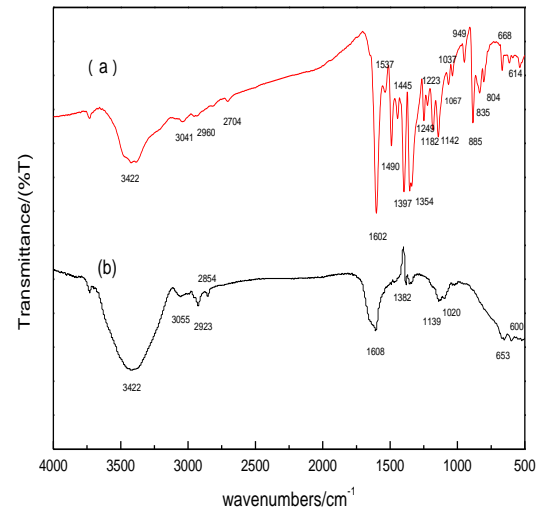

Fig.4 The FT-IR spectra of pretreatment methylene blue in photo-catalytic oxidation process

The transformation of the methylene blue molecular structure was further evaluated by measurement of the FTIR spectra at 0 and $30 \mathrm{~min}$. The results shown in Fig. 4(a) are the FTIR spectra of methylene blue solution without Photocatalytic oxidation and Fig. 4(b) displays FTIR spectra of methylene blue after Photocatalytic oxidation for $30 \mathrm{~min}$. According to the UV-Vis absorption spectra of methylene blue, it was illustrated that methylene blue had N-S heterocycle group conjugate structure and phenothiazine structure and aromatics. The results in Fig. 3(a) showed that the broad peak of $3422 \mathrm{~cm}^{-1}$ was determined as the $\mathrm{O}-\mathrm{H}$ stretching vibration of water molecules. It could be seen the peak corresponding to $\mathrm{C}-\mathrm{H}$ absorption of benzene ring occured at $3041 \mathrm{~cm}^{-1}$. The peak of $2960 \mathrm{~cm}^{-1}$ belongs to the stretching vibration peak of methyl group and the other typical flexural vibration of methyl group occurs at 1397 $\mathrm{cm}^{-1}$. The $\mathrm{C}=\mathrm{C}$ framework vibration of benzene ring vibration and the $\mathrm{C}=\mathrm{N}$ stretching vibration were found at $1602 \mathrm{~cm}^{-1[12]}$, the absorption peaks at and $1490 \mathrm{~cm}^{-1}$ and $1445 \mathrm{~cm}^{-1}$ were also related to $\mathrm{C}=\mathrm{C}$ stretchings. The characteristic absorption peak of $\mathrm{C}-\mathrm{N}$ bond linked aromatic amine occurs at $1354 \mathrm{~cm}^{-1}$. The peak of $1250 \mathrm{~cm}^{-1}$ is the $\mathrm{C}=\mathrm{S}$ stretching vibration absorption peak. The absorption peaks at $1222 \mathrm{~cm}^{-1} 、 1181 \mathrm{~cm}^{-1} 、 1143 \mathrm{~cm}^{-1}$, $1066 \mathrm{~cm}^{-1}$ and $1037 \mathrm{~cm}^{-1}$ are assigned to the in-plane bending vibration peaks of aromatic $\mathrm{C}-\mathrm{H}$, while the absorption peaks at $885 \mathrm{~cm}^{-1}, 836 \mathrm{~cm}^{-1}$ and $667 \mathrm{~cm}^{-1}$ are attributed to out-plane bending vibration peaks of aromatic C-H. The absorption peak of $614 \mathrm{~cm}^{-1}$ is caused by C-S-C framework vibration ${ }^{[13]}$.

As shown in Fig.3b, the number of absorption peaks had decreased significantly and the characteristic peak of $\mathrm{C}=\mathrm{N}$ and $\mathrm{C}=\mathrm{S}$ had decreased significantly after photocatalytic oxidation, thus it could be concluded that the N-S heterocyclic compound was broken during the degradation process. The characteristic peak of benzene and $\mathrm{C}-\mathrm{H}$ bending vibration of aromatic $\mathrm{C}-\mathrm{H}$ had fallen substantially indicating that phenyl structure was destroyed. Owing to the ring opening reaction of benzene and the rupture of dye molecules, the absorption peaks at $2923 \mathrm{~cm}^{-1}$ which belonged to the stretching vibration peak of methyl group became stronger. Thus, it is summarized that the conjugate structure of N-S heterocyclic compound was broken and aromatic ring was oxidized to open the ring, the dye molecules were degraded and formed micro-molecular organics during photo catalysis reaction. 


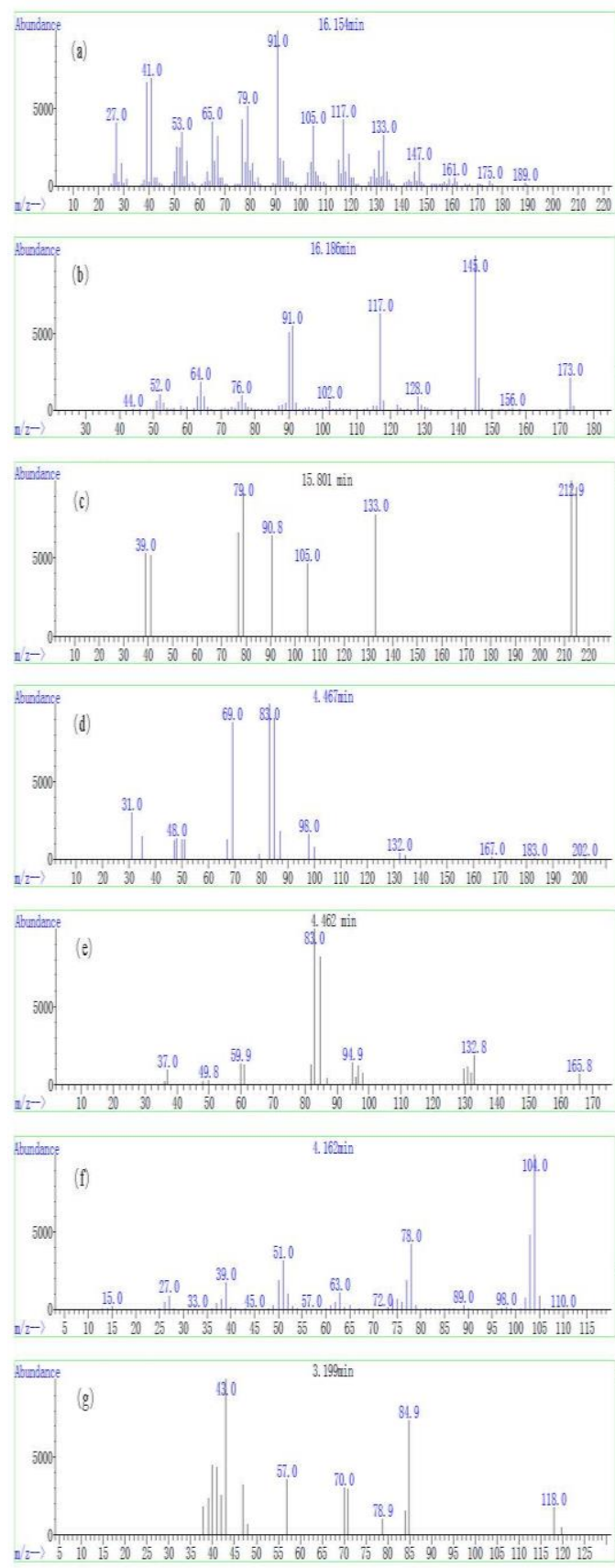

Fig.5 The GC-MS spectra of identified compounds in methylene blue solution of Photocatalytic oxidation:(a) Product at $16.186 \mathrm{~min}$, (b) Product at $16.154 \mathrm{~min}$, (c) Product at $15.801 \mathrm{~min}$, (d) Product at $4.467 \mathrm{~min}$, (e) Product at $4.462 \mathrm{~min}$, (f) Product at $4.162 \mathrm{~min},(\mathrm{~g})$ Product at $3.199 \mathrm{~min}$

In order to verify the above deductions, a further attempt was made to identify the mineralization during the degradation of methylene blue through GC-MS analyses. The qualitative results of the GC-MS analysis in Fig.5 showed that some volatile intermediate products of methylene blue solution were identified during Photocatalytic oxidation. The $\mathrm{m} / \mathrm{z}$ at 215.0 could be easily identified to 2-Amino-5-dimethylamino-benzenesulfonic acid anion (Fig. 5c) with the molecular formula being $\mathrm{C}_{8} \mathrm{H}_{11} \mathrm{~N}_{2} \mathrm{SO}_{3}$ speculated from the ion abundance ratios which suggested that N-S heterocyclic compound of methylene blue were broken. The $\mathrm{m} / \mathrm{z}$ value of 173.0 and 189.0 corresponding to 4-Amino-benzenesulfonic acid and 2-Amino-5-hydroxy-benzenesulfonic acid were likely to be the degradation intermediate of 2-Amino-5-dimethylamino-benzenesulfonic acid attacked by hydroxyl radical, whose molecular formula were $\mathrm{C}_{6} \mathrm{H}_{7} \mathrm{NSO}_{3}$ (Fig. 5a) and $\mathrm{C}_{6} \mathrm{H}_{7} \mathrm{NSO}_{4}$ (Fig. 5c) respectively. At the same time, the molecular ion peak of $\mathrm{m} / \mathrm{z} 202.0$ corresponding to 4-Nitro-benzenesulfonic acid anion was the oxidized resultant of 4-Amino-benzenesulfonic with the molecular formula being $\mathrm{C}_{8} \mathrm{H}_{10} \mathrm{~N}_{2} \mathrm{O}_{2}$ (Fig. 5d). The molecular ion peak of $\mathrm{m} / \mathrm{z} \quad 165.8$ related to Dimethyl-(4-nitro-phenyl)-amine with the molecular formula being $\mathrm{C}_{6} \mathrm{H}_{10} \mathrm{~N}_{2} \mathrm{O}_{2}$ (Fig. 5e) was the products after hydroxyl radical attacked the N-S heterocyclic of dye molecules, while the $\mathrm{m} / \mathrm{z}$ value observed at 110.0 belonging to $\mathrm{p}$-Dihydroxybenzene with the molecular formula being $\mathrm{C}_{6} \mathrm{H}_{6} \mathrm{O}_{2}$ (Fig. 5f) was the oxidized resultant of benzene ring. The $\mathrm{m} / \mathrm{z}$ value of 118.0 belonged to the small molecular products of degradation with the molecular formula being $\mathrm{C}_{4} \mathrm{H}_{6} \mathrm{O}_{4}$ (Fig.5g), corresponding to succinic acid generated by open-loop benzene. It further showed that the broken position of methylene blue treated by photocatalytic oxidation was the conjugate structure of the N-S heterocycle group which is in line with the analysis results by the UV and IR spectrogram. Based on the intermediate and above analytical results, the degradation pathways of methylene blue is described, as shown in Fig. 5. In the process of photocatalytic oxidation treatment of methylene blue, hydroxyl radicals firstly attacked the N-S heterocycle group conjugate structure because the electron density of sulfhydryl was higher, the methylene blue had been degraded to 2-Amino-5-dimethylamino-benzenesulfonic acid anion and Dimethyl-(4-nitro-phenyl)-amine. And then the Dimethyl-(4-nitro-phenyl)-amine was captured by hydroxyl radicals and formed p-Dihydroxybenzene. Furthermore, 2-Amino-5-dimethylamino-benzenesulfonic acid anion continued to be broken into 4-Amino-benzenesulfonic acid and 2-Amino-5-dimethylamino-benzenesulfonic acid. 4-Amino-benzenesulfonic acid was oxidized to 4-Nitro-benzenesulfonic acid anion. Finally, 4-Nitro-benzenesulfonic acid anion was directly attacked and generated then continued to be degraded $\mathrm{CO}_{2}$ and $\mathrm{H}_{2} \mathrm{O}$ through a series of reactions. This is similar with the degradation mechanism of methylene blue by atmospheric pressure dielectric barrier discharge plasma $^{[14]}$.

Fig.6 The degradation pathways of methylene blue reacting with Photocatalytic oxidation. 


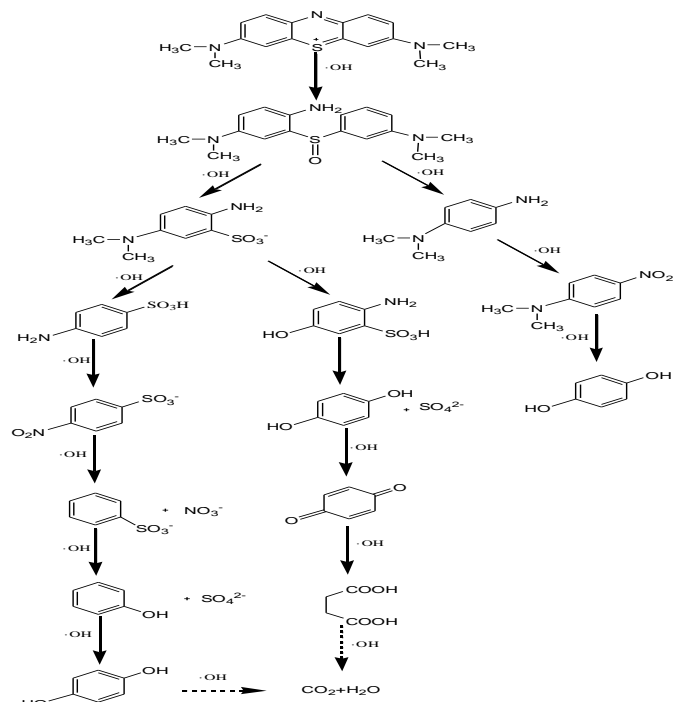

Fig.6 The degradation pathways of methylene blue reacting with Photocatalytic oxidation.

\section{Conclusions}

(1)The crystalline of $\mathrm{TiO}_{2}$ in $\mathrm{GR} / \mathrm{TiO}_{2}$ prepared by hydrothermal method is anatase and the introduction of graphene oxide into the preparation system could enhance the photocatalytic activity because of loose agglomeration and favorable dispersibility which increasing the contact area of dye molecules and catalysts.

(2)The results obtained from the variety of spectrums and chromatograms shows that the degradation of $\mathrm{MB}$ solution is that hydroxyl radicals firstly attacked the N-S heterocycle chromophore group, then the resultants were oxidized and mineralized into small-molecule products which continued to be degraded $\mathrm{CO}_{2}$ and $\mathrm{H}_{2} \mathrm{O}$ through a series of reactions.

\section{Reference}

[1]Doğan M, Özdemir Y, Alkan M. Adsorption kinetics and mechanism of cationic methyl violet and methylene blue dyes onto sepiolite[J]. Dyes and Pigments, 2007, 75(3): 701-713.

[2]Hashimoto $\mathrm{K}$, Irie $\mathrm{H}$, Fujishima A. $\mathrm{TiO}_{2}$ photocatalysis: a historical overview and future prospects[J]. Japanese journal of applied physics, 2005, 44(12R): 8269.

[3] Kumar S G, Devi L G. Review on modified $\mathrm{TiO}_{2}$ photocatalysis under UV/visible light: selected results and related mechanisms on interfacial charge carrier transfer dynamics [J]. The Journal of Physical Chemistry A, 2011, 115(46): 13211-13241.

[4]Irie H, Watanabe Y, Hashimoto K. Carbon-doped anatase $\mathrm{TiO}_{2}$ powders as a visible-light sensitive photocatalyst[J]. Chemistry Letters, 2003, 32(8): 772-773.

[5]Zaleska A. Doped-TiO 2 : a review[J]. Recent Patents on Engineering, 2008, 2(3): 157-164.

[6] Geim A K, Novoselov K S. The rise of graphene[J]. Nature materials, 2007, 6(3): 183-191.

[7] Neto A H C, Guinea F, Peres N M R, et al. The electronic properties of graphene $[\mathrm{J}]$. Reviews of modern physics, 2009, 81(1): 109.

[8]Doğan M, Abak H, Alkan M. Adsorption of methylene blue onto hazelnut shell: Kinetics, mechanism and activation parameters $[\mathrm{J}]$. Journal of Hazardous Materials, 2009, 164(1): 172-181.

[9]Özer D, Dursun G, Özer A. Methylene blue adsorption from aqueous solution by dehydrated peanut hull[J]. Journal of Hazardous Materials, 2007, 144(1): 1

[10]Tayade R J, Natarajan T S, Bajaj H C. Photocatalytic degradation of methylene blue dye using ultraviolet light emitting diodes[J]. Industrial \& Engineering Chemistry Research, 2009, 48(23): 10262-10267.

[11] Hummers Jr W S, Offeman R E. Preparation of graphitic oxide $[\mathrm{J}]$. Journal of the American Chemical Society, 1958, 80(6): 1339-1339.

[12]Imamura K, Ikeda E, Nagayasu $T$, et al. Adsorption behavior of methylene blue and its congeners on a stainless steel surface[J]. Journal of colloid and interface science, 2002, 245(1): 50-57.

[13]Xiao G N, Man S Q. Surface-enhanced Raman scattering of methylene blue adsorbed on cap-shaped silver nanoparticles[J]. Chemical Physics Letters, 2007, 447(4): 305-309.

[14]Huang F, Chen L, Wang H, et al. Analysis of the degradation mechanism of methylene blue by atmospheric pressure dielectric barrier discharge plasma[J]. Chemical Engineering Journal, 2010, 162(1): 250-256. 